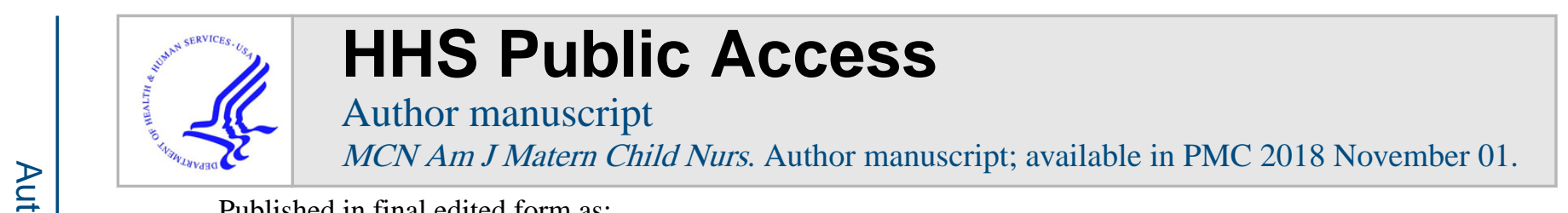

Published in final edited form as:

MCN Am J Matern Child Nurs. 2017 ; 42(6): 310-317. doi:10.1097/NMC.0000000000000372.

\title{
The Maternal Gut Microbiome during Pregnancy
}

\author{
Sara M. Edwards, CNM, MN, MPH [PhD Candidate, Instructor], \\ Laney Graduate School and Nell Hodgson Woodruff School of Nursing, Emory University
}

Solveig A. Cunningham, PhD [Assistant Professor], Rollins School of Public Health, Emory University

Anne L. Dunlop, MD, MPH, FAAFP [Associate Research Professor], and Nell Hodgson Woodruff School of Nursing, Emory University

Elizabeth J. Corwin, PhD, RN, FAAN [Professor, Associate Dean for Research] Nell Hodgson Woodruff School of Nursing, Emory University

\section{Abstract}

The gut microbiome is a critical component of an individual's metabolism and overall health. The prenatal period is marked by unique inflammatory and immune changes that alter maternal gut function and bacterial composition as the pregnancy advances. The composition of the maternal gut microbiome contributes to obstetric outcomes with long-term health sequelae for mother and child. Estrogen and progesterone also impact gut function, especially during the prenatal period. These physiologic changes in pregnancy allow for adjustments in maternal metabolism and weight necessary to support the pregnancy. This article will review the normal hormonal, metabolic and immunologic changes to the maternal gut microbiome throughout the prenatal period, in addition to relevant implications for nurses providing care for pregnant women.

\section{Keywords}

Gastrointestinal Microbiome; Microbiota; Pregnancy; Maternal Health

\section{An Overview of the Gut Microbiome}

The 100 trillion bacteria that reside in the human intestinal tract, referred to as the gut microbiota, are essential to human metabolism and immunity (Power, O'Toole, Stanton, Ross, \& Fitzgerald, 2014) (see Table 1 for definitions of terms used in this article). The most recent estimate of the ratio of bacteria to human cells in the adult body is approximately 1:1 (Sender, Fuchs, \& Milo, 2016). It is also estimated that the genes contained within the human microbiota, known as the microbiome, are 150-fold greater than those contained within the individual human genome (Power et al., 2014). The development of the human gut microbiome begins before birth and proceeds in a systematic manner, affected by factors including maternal oral microbiome, mode of delivery, maternal and infant diet, and

Conflicts of Interest

The authors report no conflicts of interest exist 
environmental exposures (Brown et al., 2013). Development of the microbiome occurs simultaneously with, and plays a key role in neurologic system maturation (Borre et al., 2014). Significant early-life disruptions of the microbiome, such as through major illness or exposure to high dose, broad-spectrum antibiotics, can lead to chronic disease or mental disorders later in life (Borre et al., 2014). While the entire gut begins in the mouth and continues to the anus, the gut microbiome describes the intestinal portion alone, given that the intestine harbors the most diverse and abundant microbial community in the body (Backhed et al., 2012). The focus of this review, therefore, is the gut microbiome as it is commonly defined, limited to the intestines. More specifically, the state of the science will then be presented regarding the maternal gut during pregnancy, related maternal-fetal health implications and evidence-based nursing care strategies to improve delivery of care and optimize health.

The gut microbiome provides a broad range of functions. Most importantly, it collects indigestible particles from food into fecal waste and assimilates nutritional particles, such as vitamins and minerals (Mayer, Savidge, \& Shulman, 2014). It acts with the liver to detoxify and excrete xenobiotics, ubiquitous harmful foreign chemicals present in the environment (Mayer, Savidge, et al., 2014). An individual's systemic and intestinal immune systems depend greatly on the gut microbiome's function of epithelial cell renewal and management of intestinal integrity (Power et al., 2014). Without a healthy intestinal wall, bacteria migrate across the gut into the general circulation increasing systemic inflammation (Power et al., 2014). This migration occurs through a mechanism of intestinal permeability sometimes referred to as "leaky gut" (Power et al., 2014). With leaky gut, junctions, composed of various proteins that link adjacent intestinal epithelial cells, are weak and fail to act as an integral barrier to migrating pathogens (Power et al., 2014). Increased intestinal permeability is hypothesized to be the mechanism by which a dysbiotic gut is linked with low-grade inflammatory disorders like obesity and its associated conditions, insulin-resistance and Type 2 diabetes mellitus (Mokkala et al., 2016). High permeability allows the leakage of bacteria, and/or bacterial components like the lipopolysaccharides (LPS) contained in the cell walls of gram-negative bacteria, thus inducing a deleterious systemic inflammatory condition called metabolic endotoxemia (Cani, Osto, Geurts, \& Everard, 2012).

\section{Immunologic Changes of the Gut Microbiome in Pregnancy}

During pregnancy, inflammatory states vary from higher inflammation at implantation and in labor to lower levels of inflammation in mid-pregnancy (Trevisanuto et al., 2013). Antiinflammatory properties exist at the placental bed which protects the fetus from rejection, yet the mucosal surfaces of the gut and other tissues experience a low-grade inflammation with rising levels of pro-inflammatory cytokines and white cells as the pregnancy advances (Koren et al., 2012). When conditions like maternal obesity, gestational diabetes or a leaky gut shift the maternal inflammatory state from a physiologic to an excessive level, vascular dysfunction of the placental tissue can develop leading to deleterious effects such as fetal growth restriction and preeclampsia (Kashtanova et al., 2016; Kim, Romero, Chaemsaithong, \& Kim, 2015). Gut microbes found in amniotic fluid of women who experienced preterm premature rupture of membranes suggest that the gut microbiome could play a role in intrauterine infections (DiGiulio et al., 2010). Possible mechanisms of 
microbial translocation have been proposed; either by lipopolysaccharides (LPS) from gram negative bacteria that increase inflammatory mediators and prostaglandin production, via ascending inoculation through the vagina, or hematogenous spread of microbes from a leaky gut to the placenta or uterus (Nuriel-Ohayon, Neuman, \& Koren, 2016).

\section{Factors That Can Modify the Gut Microbiome}

The gut microbiome is also shaped by diet and genetics. The typical Western diet consists of excessive processed foods, dietary fat and sugars (Morrison \& Regnault, 2016). Such a diet promotes excess weight gain and a dysbiotic gut and is associated with adverse maternal and child health outcomes (Dunlop et al., 2015; Morrison \& Regnault, 2016). Conversely, certain dietary nutrients, namely low-fat protein (for instance beans, skinless chicken, lean beef), organic proteins and produce (which reduces exposure to dietary antibiotics and pesticides), unsaturated fatty acids (for instance in canola and olive oils, flaxseeds, and salmon), whole grains, and certain strains of probiotics have been found to promote a healthy gut microbiome, enhance intestinal integrity and reduce excessive systemic inflammation (Griffin, 2015; Kashtanova et al., 2016). Short chain fatty acids (SCFA), such as acetate, propionate and butyrate, are byproducts of fiber fermentation called prebiotics and serve as an energy source to intestinal epithelial cells and enhance the connections between these cells (David et al., 2014). SCFA production by the gut microbiota is negatively correlated with body mass index (Gomez-Arango et al., 2016). Increased maternal serum SCFA levels can positively influence metabolic changes seen in pregnancy: maternal weight gain, glucose metabolism and levels of various metabolic hormones (Koren et al., 2012; Priyadarshini et al., 2014). A recent study to evaluate the relationships among maternal weight, gut microbiome, blood pressure and plasminogen activator inhibitor-1 (PAI-1) levels in overweight and obese pregnant women found the abundance of butyrate-producing bacteria to be significantly negatively associated with systolic and diastolic blood pressure and PAI-1 levels (Gomez-Arango et al., 2016). These findings suggest increasing butyrate-producing bacteria may contribute to lower BP in overweight and obese pregnant women. Studies of omega-3 long-chain polyunsaturated fatty acids (PUFAs) also suggest protection of the intestinal wall through strengthening of cellular connections (Li et al., 2008). A recent clinical trial examined the effect of $2000 \mathrm{mg}$ omega-3 supplementation versus placebo in overweight and obese pregnant women (Haghiac et al., 2015). While the gut microbiome was not evaluated, the primary finding was a significant reduction in the plasma inflammatory marker CRP among the supplemented group (Haghiac et al., 2015). This could prove to be a strategy to reduce the low grade inflammation and higher obstetric risks suffered by overweight and obese women or those with gestational diabetes.

\section{The Brain-Gut Axis}

Animal and human studies support the existence of a significant bidirectional relationship between the gut and the brain, involving multiple neurologic and endocrine signaling systems (Mayer, Knight, Mazmanian, Cryan, \& Tillisch, 2014). Normal central nervous system function relies on a healthy gut microbiome or "eubiosis" (Aitken \& Gewirtz, 2013). The discovery of two-way signaling between the gut and brain has led to this system being coined, the "Brain-Gut Axis" (Foster \& McVey Neufeld, 2013; Gareau, 2014). In a healthy 
individual, the autonomic nervous system (ANS), the enteric nervous system (ENS), the hypothalamic-pituitary-adrenal (HPA) axis, and the immune system work in synchrony bidirectionally between brain and gut (Cepeda, Katz, \& Blacketer, 2016; Foster \& McVey Neufeld, 2013). When there is a disruption of any of the components of this system, disease may manifest (Foster \& McVey Neufeld, 2013; Gareau, 2014). The bidirectional nature of the brain-gut axis, magnified by gut inflammation, can impact the central nervous system, body weight, immune status and behavior (Cepeda et al., 2016) (see Figure 1).

The impact of stress on the neuroendocrine system, especially when chronic, can result in immunologic dysregulation and heightened risk and virulence of infection (Cepeda, Katz, \& Blacketer, 2016). Disproportionate rates of infections have been found in chronically stressed minority populations and are thought to be a source of higher rates of preterm birth in these populations through dysbiotic gut microbe translocation mechanisms previously described (Culhane, Rauh, \& Goldenberg, 2006; Mendez, Hogan, \& Culhane, 2014).

\section{Hormonal Changes in Pregnancy and the Gut Microbiome}

The prenatal period is marked by dramatic hormonal shifts with resultant unique inflammatory and immune changes that alter gut function and bacterial composition (Brantsaeter et al., 2011; Koren et al., 2012). Estrogen and progesterone impact the composition of the gut microbiome through their effect on bacterial metabolism and growth and virulence of pathogenic bacteria (Mulak et al., 2014). An example of this is the susceptibility to Listeria monocytogenes infections in pregnancy partly due to elevated estrogen and progesterone levels, leading to adverse outcomes, including preterm delivery or stillbirth (Garcia-Gomez, Gonzalez-Pedrajo, \& Camacho-Arroyo, 2013). Dramatic changes in ovarian hormones during the prenatal and postpartum periods also affect gut contractility and transit (Mayer, Savidge, et al., 2014). It is plausible that the hormone levels involved in increasing gut transit time are an adaptive response to allow greater nutrient and energy harvest, thus promoting weight gain in pregnancy.

\section{Metabolic Changes in Pregnancy and the Gut Microbiome}

The consequences of gut dysbiosis that lead to obesity and, likely, to excessive gestational weight gain, have only recently been explored in human pregnancy (Angelakis, Merhej, \& Raoult, 2013). Of particular interest is the recognition that the immune and metabolic changes that occur normally in pregnancy are actually comparable to metabolic syndrome outside of pregnancy (Chassaing \& Gewirtz, 2014). With no differences in diet, including total energy intake, pregnant women have been found to gain greater adiposity with significantly higher leptin, insulin and insulin resistance measures, cholesterol, and glycated hemoglobin with each trimester of pregnancy compared to their non-pregnant counterparts (Collado, Isolauri, Laitinen, \& Salminen, 2008). The microbial diversity in the gut at the start of pregnancy appears to be similar to that of non-pregnant women (Santacruz et al., 2010). Yet, as the pregnancy advances, the abundance of gut bacteria associated with inflammatory states increases in nearly $70 \%$ of women (Santacruz et al., 2010). The greatest change in the gut microbiota occurs in the ratio of certain key bacteria (Firmicutes: Bacteroidetes), mimicking the higher levels of Firmicutes seen in obesity (Santacruz et al., 
2010). Levels of proinflammatory cytokines (including IFN-y, IL-2, IL-6 and TNF-a) also rise in serum; adipose and placental tissue later in pregnancy and the mucosal surfaces throughout the gastrointestinal tract reflect a low-grade inflammatory state (Cani et al., 2012). Thus, while these same metabolic and immunologic changes in a man or nonpregnant woman would be considered abnormal and a sign of a disease state, in the context of a normal pregnancy, they appear to be required, improving energy storage in fat and providing for fetal growth and lactation (Cani et al., 2012; Chassaing \& Gewirtz, 2014).

\section{Clinical Implications of the Gut Microbiome in Pregnancy}

The gut microbiome and the brain-gut axis are key players in the critical prenatal period when the maternal and fetal microbiome are particularly sensitive and changes can impact fetal brain development (Borre et al., 2014; Mayer, Knight, et al., 2014). Negative interference with the brain-gut axis (depicted on right side of Figure 1) during these times is associated with a higher maternal and child risk for chronic intestinal disorders, conditions which affect weight and growth, and even neuropsychiatric disorders (Mayer, Knight, et al., 2014). Health care recommendations that promote the IOM guidelines for maternal weight gain by prepregnant BMI category, high quality diet and gut eubiosis should be considered in the delivery of prenatal care and in client education. Stress, infection and antibiotic use, especially during pregnancy, can lead to dysbiosis and may increase risk of maternal and infant neurodevelopmental disorders (Bilder et al., 2013; Vela et al., 2015). Appropriate type, duration and indication of prebiotic, probiotic and antibiotic use in the prevention and treatment of illness will become an important topic for discussion with clients in the future, since all act on the gut microbiome and may impact overall health and gestational weight gain (Griffin, 2015). There are no current clinical guidelines to facilitate these discussions pending further research.

Dietary and lifestyle patterns that impact the brain-gut axis also need to be assessed. When a mother gains excessive gestational weight or enters pregnancy obese, metabolic changes in pregnancy are magnified, and thus the risk of gestational diabetes, fetal macrosomia, or preeclampsia is elevated (Josefson, Hoffmann, \& Metzger, 2013). Identification of food sensitivities (gluten, dairy, and nuts are common) and elimination of those foods from the diet reduce systemic inflammation and improves gut health. Pre- and probiotic use and increasing dietary fiber and fermented foods rich in probiotics and enzymes (such as yogurt, kefir, and miso) promotes eubiosis of the gut, possibly reducing risk of elevated blood pressure and improving metabolic parameters in vulnerable overweight and obese pregnant women. Further, fiber can improve constipation and other gastrointestinal conditions common in pregnancy (Griffin, 2015). Primary care and preconception evaluations should also include dietary and lifestyle assessment and counseling to reach a healthy prepregnant body mass index and optimize overall health (Egan et al., 2014).

In a recent clinical trial, supplementation with specific strains of probiotics, such as Lactobacillus rhamnosus GG and Lactobacillus acidophilus, combined with dietary counseling have been found to improve glucose metabolism in healthy pregnant and lactating women (Brantsaeter et al., 2011). Daily intake of milk-based dietary probiotics has been reported to reduce blood pressure in pregnancy as well as the incidence of 
preeclampsia among primiparous women (Brantsaeter et al., 2011; Gomez-Arango et al., 2016). Lactose intolerance can often be managed by ingestion of lactase enzymes with the dairy or by limiting intake to lactose-free dairy products. Daily yogurt consumption containing $L$. rhamnosus was found to have a statistically significant protective effect against heavy metal toxicity (mercury and arsenic) in pregnant women with high risk diets (Bisanz et al., 2014). Probiotic milk and other food products may, therefore, be worth consideration for women at greater risk for gestational diabetes, preeclampsia and some environmental toxins.

\section{Conclusion}

Diet and lifestyle practices are modifiable factors that can affect the brain-gut axis, and ultimately, the long-term health of women and infants. The degree to which the gut microbiome contributes to the neurodevelopmental, immunological and intestinal health of the pregnant woman and her fetus is only now beginning to be measured. Health practices and novel therapies to promote eubiosis and treat dysbiosis of the maternal gut are still to be discovered but will likely prove fundamental in the care of women in pregnancy.

\section{Acknowledgments}

Sources of Funding

Funding Sources: NIH F31NR015722, NIH R01-NR014800

\section{References}

Aitken JD, Gewirtz AT. Gut microbiota in 2012: Toward understanding and manipulating the gut microbiota. Nature Reviews Gastroenterology \& Hepatology. 2013; 10:72-74. DOI: 10.1038/ nrgastro.2012.252 [PubMed: 23296245]

Angelakis E, Merhej V, Raoult D. Related actions of probiotics and antibiotics on gut microbiota and weight modification. The Lancet Infectious Diseases. 2013; 13:889-899. DOI: 10.1016/ s1473-3099(13)70179-8 [PubMed: 24070562]

Backed F, Fraser CM, Ringel Y, Sanders ME, Sartor RB, Sherman PM, Finlay BB. Defining a healthy human gut microbiome: current concepts, future directions, and clinical applications. Cell Host Microbe. 2012; 12(5):611-622. DOI: 10.1016/j.chom.2012.10.012 [PubMed: 23159051]

Bilder DA, Bakian AV, Viskochil J, Clark EA, Botts EL, Smith KR, ... Coon H. Maternal prenatal weight gain and autism spectrum disorders. Pediatrics. 2013; 132(5):e1276-1283. DOI: 10.1542/ peds.2013-1188 [PubMed: 24167172]

Bisanz JE, Enos MK, Mwanga JR, Changalucha J, Burton JP, Gloor GB, Reid G. Randomized openlabel pilot study of the influence of probiotics and the gut microbiome on toxic metal levels in Tanzanian pregnant women and school children. mBio. 2014; 5(5):e01580-01514. DOI: 10.1128/ mBio.01580-14 [PubMed: 25293764]

Borre YE, O'Keeffe GW, Clarke G, Stanton C, Dinan TG, Cryan JF. Microbiota and neurodevelopmental windows: Implications for brain disorders. Trends in Molecular Medicine. 2014; 20:509-518. DOI: 10.1016/j.molmed.2014.05.002 [PubMed: 24956966]

Brantsaeter AL, Myhre R, Haugen M, Myking S, Sengpiel V, Magnus P, ... Meltzer HM. Intake of probiotic food and risk of preeclampsia in primiparous women: The Norwegian Mother and Child Cohort Study. American Journal of Epidemiology. 2011; 174:807-815. DOI: 10.1093/aje/kwr168 [PubMed: 21821542]

Brown J, de Vos WM, DiStefano PS, Dore J, Huttenhower C, Knight R, ... Turnbaugh P. Translating the human microbiome. Nature Biotechnology. 2013; 31:304-308. DOI: 10.1038/nbt.2543 
Cani PD, Osto M, Geurts L, Everard A. Involvement of gut microbiota in the development of lowgrade inflammation and type 2 diabetes associated with obesity. Gut microbes. 2012; 3:279-288. DOI: 10.4161/gmic.19625 [PubMed: 22572877]

Cepeda MS, Katz EG, Blacketer C. Microbiome-Gut-Brain axis: probiotics and their association with depression. Journal of Neuropsychiatry and Clinical Neurosciences. 2016; Advance online publication. doi: 10.1176/appi.neuropsych.15120410

Culhane JF, Rauh VA, Goldenberg RL. Stress, bacterial vaginosis, and the role of immune processes. Curr Infect Dis Rep. 2006; 8(6):459-464. Retrieved from http://www.ncbi.nlm.nih.gov/pubmed/ 17064639. [PubMed: 17064639]

Chassaing B, Gewirtz AT. Gut microbiota, low-grade inflammation, and metabolic syndrome. Toxicologic Pathology. 2014; 42:49-53. DOI: 10.1177/0192623313508481 [PubMed: 24285672]

Collado MC, Isolauri E, Laitinen K, Salminen S. Distinct composition of gut microbiota during pregnancy in overweight and normal-weight women. American Journal of Clinical Nutrition. 2008; 88:894-899. [PubMed: 18842773]

David LA, Maurice CF, Carmody RN, Gootenberg DB, Button JE, Wolfe BE, ... Turnbaugh PJ. Diet rapidly and reproducibly alters the human gut microbiome. Nature. 2014; 505:559-563. DOI: 10.1038/nature12820 [PubMed: 24336217]

DiGiulio DB, Romero R, Kusanovic JP, Gomez R, Kim CJ, Seok KS, ... Relman DA. Prevalence and diversity of microbes in the amniotic fluid, the fetal inflammatory response, and pregnancy outcome in women with preterm pre-labor rupture of membranes. American Journal of Reproductive Immunology. 2010; 64(1):38-57. DOI: 10.1111/j.1600-0897.2010.00830.x [PubMed: 20331587]

Dunlop AL, Mulle JG, Ferranti EP, Edwards S, Dunn AB, Corwin EJ. Maternal Microbiome and Pregnancy Outcomes That Impact Infant Health: A Review. Adv Neonatal Care. 2015; 15(6):377385. DOI: 10.1097/ANC.0000000000000218 [PubMed: 26317856]

Egan AM, Dennedy MC, Al-Ramli W, Heerey A, Avalos G, Dunne F. ATLANTIC-DIP: Excessive gestational weight gain and pregnancy outcomes in women with gestational or pregestational diabetes mellitus. The Journal of Clinical Endocrinology and Metabolism. 2014; 99:212-219. DOI: 10.1210/jc.2013-2684 [PubMed: 24187402]

Foster JA, McVey Neufeld KA. Gut-brain axis: How the microbiome influences anxiety and depression. Trends in Neurosciences. 2013; 36:305-312. DOI: 10.1016/j.tins.2013.01.005 [PubMed: 23384445]

Garcia-Gomez E, Gonzalez-Pedrajo B, Camacho-Arroyo I. Role of sex steroid hormones in bacterialhost interactions. Biomed Research International. 2013; 2013:1-10. DOI: 10.1155/2013/928290

Gareau MG. Microbiota-gut-brain axis and cognitive function. Advances in Experimental Medicine and Biology. 2014; 817:357-371. DOI: 10.1007/978-1-4939-0897-4_16 [PubMed: 24997042]

Gomez-Arango LF, Barrett HL, McIntyre HD, Callaway LK, Morrison M, Dekker Nitert M. Connections Between the Gut Microbiome and Metabolic Hormones in Early Pregnancy in Overweight and Obese Women. Diabetes. 2016; 65(8):2214-2223. DOI: 10.2337/db16-0278 [PubMed: 27217482]

Gomez-Arango LF, Barrett HL, McIntyre HD, Callaway LK, Morrison M, Dekker Nitert M. Increased systolic and diastolic blood pressure is associated with altered gut microbiota composition and butyrate production in early pregnancy. Hypertension. 2016; 68:974-981. DOI: 10.1161/ hypertensionaha.116.07910 [PubMed: 27528065]

Griffin C. Probiotics in obstetrics and gynaecology. Australian and New Zealand Journal of Obstetrics and Gynaecology. 2015; 55:201-209. DOI: 10.1111/ajo.12303 [PubMed: 26052924]

Haghiac M, Yang XH, Presley L, Smith S, Dettelback S, Minium J, ... Hauguel-de Mouzon S. Dietary Omega-3 Fatty Acid Supplementation Reduces Inflammation in Obese Pregnant Women: A Randomized Double-Blind Controlled Clinical Trial. PloS one. 2015; 10(9):e0137309.doi: 10.1371/journal.pone.0137309 [PubMed: 26340264]

Josefson JL, Hoffmann JA, Metzger BE. Excessive weight gain in women with a normal prepregnancy BMI is associated with increased neonatal adiposity. Pediatric Obesity. 2013; 8:e33-36. DOI: 10.1111/j.2047-6310.2012.00132.x [PubMed: 23283756] 
Kashtanova DA, Popenko AS, Tkacheva ON, Tyakht AB, Alexeev DG, Boytsov SA. Association between the gut microbiota and diet: Fetal life, early childhood, and further life. Nutrition. 2016; 32:620-627. DOI: 10.1016/j.nut.2015.12.037 [PubMed: 26946974]

Kim CJ, Romero R, Chaemsaithong P, Kim JS. Chronic inflammation of the placenta: definition, classification, pathogenesis, and clinical significance. American Journal of Obstetrics and Gynecology. 2015; 213:S53-69. DOI: 10.1016/j.ajog.2015.08.041 [PubMed: 26428503]

Koren O, Goodrich JK, Cullender TC, Spor A, Laitinen K, Backhed HK, ... Ley RE. Host remodeling of the gut microbiome and metabolic changes during pregnancy. Cell. 2012; 150:470-480. DOI: 10.1016/j.cell.2012.07.008 [PubMed: 22863002]

Laitinen K, Poussa T, Isolauri E. Probiotics and dietary counselling contribute to glucose regulation during and after pregnancy: A randomised controlled trial. The British Journal of Nutrition. 2009; 101:1679-1687. DOI: 10.1017/s0007114508111461 [PubMed: 19017418]

Li Q, Zhang Q, Wang M, Zhao S, Xu G, Li J. n-3 polyunsaturated fatty acids prevent disruption of epithelial barrier function induced by proinflammatory cytokines. Molecular Immunology. 2008; 45:1356-1365. DOI: 10.1016/j.molimm.2007.09.003 [PubMed: 17936906]

Luoto R, Laitinen K, Nermes M, Isolauri E. Impact of maternal probiotic-supplemented dietary counselling on pregnancy outcome and prenatal and postnatal growth: A double-blind, placebocontrolled study. British Journal of Nutrition. 2010; 103:1792-1799. DOI: 10.1017/ S0007114509993898 [PubMed: 20128938]

Ma J, Prince AL, Bader D, Hu M, Ganu R, Baquero K, ... Aagaard KM. High-fat maternal diet during pregnancy persistently alters the offspring microbiome in a primate model. Nature communications. 2014; 5:3889.doi: 10.1038/ncomms4889

Mayer EA, Knight R, Mazmanian SK, Cryan JF, Tillisch K. Gut microbes and the brain: Paradigm shift in neuroscience. Journal of Neuroscience. 2014; 34:15490-15496. DOI: 10.1523/ JNEUROSCI.3299-14.2014 [PubMed: 25392516]

Mayer EA, Savidge T, Shulman RJ. Brain-gut microbiome interactions and functional bowel disorders. Gastroenterology. 2014; 146:1500-1512. DOI: 10.1053/j.gastro.2014.02.037 [PubMed: 24583088]

Mendez DD, Hogan VK, Culhane JF. Institutional racism, neighborhood factors, stress, and preterm birth. Ethnicity and Health. 2014; 19(5):479-499. DOI: 10.1080/13557858.2013.846300 [PubMed: 24134165]

Morrison JL, Regnault TR. Nutrition in Pregnancy: Optimising Maternal Diet and Fetal Adaptations to Altered Nutrient Supply. Nutrients. 2016; 8(6)doi: 10.3390/nu8060342

Mokkala K, Roytio H, Munukka E, Pietila S, Ekblad U, Ronnemaa T, ... Laitinen K. Gut microbiota richness and composition and dietary intake of overweight pregnant women are related to serum zonulin concentration, a marker for intestinal permeability. The Journal of Nutrition. 2016; 146:1694-1700. DOI: 10.3945/jn.116.235358 [PubMed: 27466607]

Mulak A, Tache Y, Larauche M. Sex hormones in the modulation of irritable bowel syndrome. World Journal of Gastroenterology. 2014; 20:2433-2448. DOI: 10.3748/wjg.v20.i10.2433 [PubMed: 24627581]

Nuriel-Ohayon M, Neuman H, Koren O. Microbial Changes during Pregnancy, Birth, and Infancy. Front Microbiol. 2016; 7:1031.doi: 10.3389/fmicb.2016.01031 [PubMed: 27471494]

Power SE, O’Toole PW, Stanton C, Ross RP, Fitzgerald GF. Intestinal microbiota, diet and health. The British Journal of Nutrition. 2014; 111:387-402. DOI: 10.1017/s0007114513002560 [PubMed: 23931069]

Priyadarshini M, Thomas A, Reisetter AC, Scholtens DM, Wolever TM, Josefson JL, Layden BT. Maternal short-chain fatty acids are associated with metabolic parameters in mothers and newborns. Translational Research. 2014; 164:153-157. DOI: 10.1016/j.trsl.2014.01.012 [PubMed: 24530607]

Santacruz A, Collado MC, Garcia-Valdes L, Segura MT, Martin-Lagos JA, Anjos T, ... Sanz Y. Gut microbiota composition is associated with body weight, weight gain and biochemical parameters in pregnant women. The British Journal of Nutrition. 2010; 104:83-92. DOI: 10.1017/ s0007114510000176 [PubMed: 20205964]

Sender R, Fuchs S, Milo R. Revised Estimates for the Number of Human and Bacteria Cells in the Body. PLoS Biol. 2016; 14(8):e1002533.doi: 10.1371/journal.pbio.1002533 [PubMed: 27541692] 
Shin GH, Toto EL, Schey R. Pregnancy and postpartum bowel changes: Constipation and fecal incontinence. American Journal of Gastroenterology. 2015; 110:521-529. DOI: 10.1038/ajg. 2015.76 [PubMed: 25803402]

Trevisanuto D, Peruzzetto C, Cavallin F, Vedovato S, Cosmi E, Visentin S, ... Zanardo V. Fetal placental inflammation is associated with poor neonatal growth of preterm infants: a case-control study. The journal of maternal-fetal \& neonatal medicine: the official journal of the European Association of Perinatal Medicine, the Federation of Asia and Oceania Perinatal Societies, the International Society of Perinatal Obstetricians. 2013; 26(15):1484-1490. DOI: 10.3109/14767058.2013.789849

Vela G, Stark P, Socha M, Sauer AK, Hagmeyer S, Grabrucker AM. Zinc in gut-brain interaction in autism and neurological disorders. Neural Plasticity, 2015. 2015; doi: 10.1155/2015/972791

\section{Callouts}

Starting in the prenatal period, maternal health and diet play a critical role in the foundation of the child's gut microbiome with long-lasting health implications.

Promotion of a eubiotic gut through dietary intake of specific probiotics can improve gut health and reduce systemic inflammation.

The rise in estrogen and progesterone during pregnancy alters gut function and microbiome composition, increasing vulnerability to pathogens.

The gut microbiota progressively changes with each trimester of pregnancy; the composition is most commonly dominated by Firmicutes as in obese populations.

Nursing strategies such as the judicious use of antibiotics and personalized education regarding a healthy lifestyle can promote a client's eubiotic gut. 


\section{Clinical Implications}

Nurses can promote gut health in pregnant patients by:

- Conducting a detailed client dietary assessment, individualized education and counseling to follow a diet with 70-90 grams low fat, organic protein daily, unsaturated fatty acids, whole grains, high fiber, low processed sugars that includes daily probiotic dairy or other cultured food products (especially containing L. acidophilus, B. lactis, and L. rhamnosus).

- $\quad$ Encouraging omega-3 polyunsaturated fatty acid dietary intake, such as through ground flax seeds, wild-caught salmon and tuna (up to twice weekly) or $2000 \mathrm{mg}$ pharmaceutical grade omega-3 supplementation which can increase intestinal wall integrity, reduce inflammatory markers in serum and tissues and improve glucose metabolism.

- Assessing clinical prenatal labs that reflect gut health including normal glucose tolerance test and complete blood count.

- Identifying and treating clinical conditions that reflect gut dysbiosis including preexisting eating disorders such as obesity, insufficient or excessive weight gain; gastrointestinal disorders including hyperemesis gravidarum, cholelithiasis, gastroesophageal reflux disease; infectious conditions such as listeriosis or toxoplasmosis; and obstetric complications such as preterm labor, gestational diabetes or preeclampsia.

- $\quad$ Evaluating sources and perceived level of stress, mood, pain, sleep, and support systems utilized to help clients in identifying ways to improve the gut-brain axis (consider psychiatric or social work referral, prenatal exercise, nonpharmacologic sleep hygiene aids, or chiropractic care.)

- Monitoring gestational weight that follows the IOM guidelines to minimize the risk of metabolic and hypertensive disorders and CNS dysregulation, especially in high-risk groups. Counselling to increase dietary fiber and probiotic foods or supplements can confer additional protection in the overweight and obese woman. 


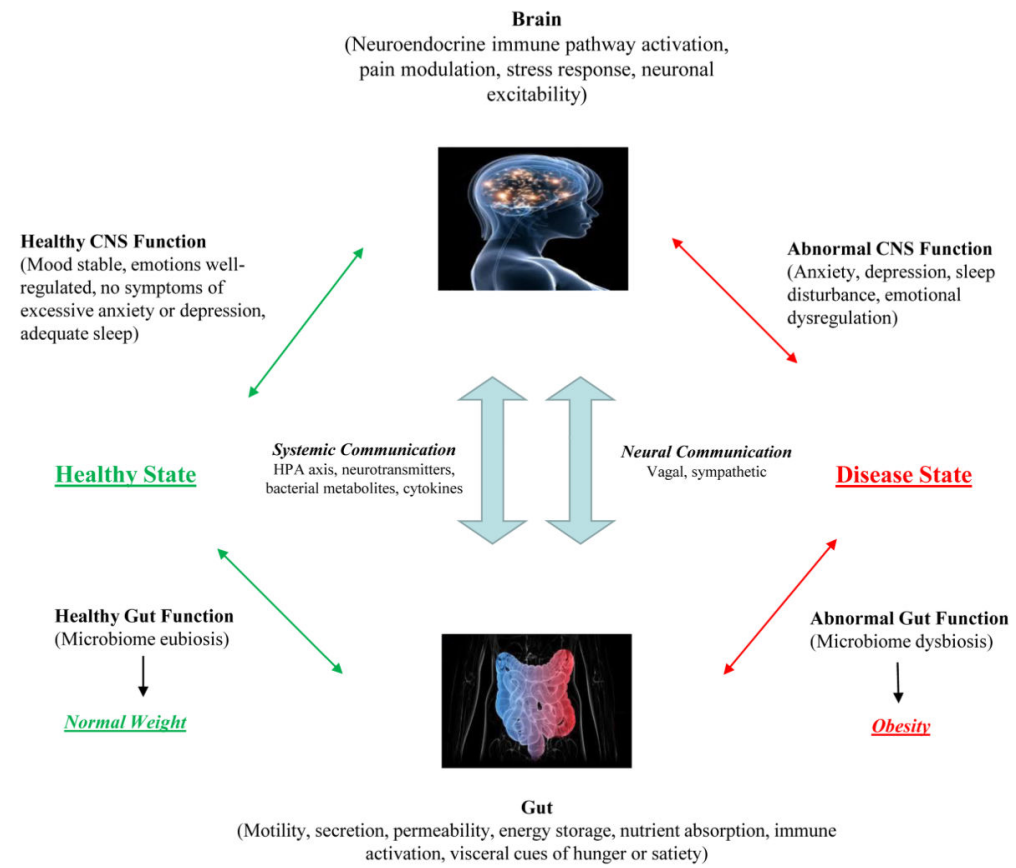

Figure 1.

The bidirectional communication of the brain-gut axis occurs via systemic and neural mechanisms. It contributes to a healthy state with gut eubiosis and normal weight or a disease state with gut dysbiosis and increased risk of obesity. 


\section{Table 1}

\section{Definition of Terms}

\begin{tabular}{ll}
\hline TERM & DEFINITION \\
\hline Antibiotic & $\begin{array}{l}\text { A type of antimicrobial drug used in the prevention or treatment of bacterial infections; some also have } \\
\text { antiprotozoal activity }\end{array}$ \\
$\begin{array}{l}\text { Brain-Gut Axis (Or Gut- } \\
\text { Microbiome-Brain Axis) }\end{array}$ & $\begin{array}{l}\text { Bidirectional signaling between the gut microbes and the brain via immunological, endocrine, and neural } \\
\text { pathways }\end{array}$ \\
Dysbiosis & An unhealthy imbalance in microbial composition in a part of the body \\
Eubiosis & A microbial composition that is balanced and associated with health in a part of the body \\
Microbial Diversity & $\begin{array}{l}\text { The range of different kinds of unicellular organisms, bacteria, archaea, protists, and fungi in a particular } \\
\text { environment } \\
\text { The microbial community that resides on or within a particular environment, including bacteria, archaea, } \\
\text { protists, and fungi }\end{array}$ \\
Prebiotic & $\begin{array}{l}\text { Selectively fermented dietary ingredients that result in specific changes in the consumption and/or activity of } \\
\text { the gastrointestinal microbiota }\end{array}$ \\
Probiotic & Live microorganisms which when administered in adequate amounts confer a health effect on the host
\end{tabular}

